function $(H A Q<0.5)$ was achieved in $33 \%(530 / 1590)$ of all patients. Autoantibody-positivity was also not associated with $\mathrm{HAQ}<0.5$ (HR1.05, $95 \% \mathrm{Cl}$ $0.71 ; 1.57)$.

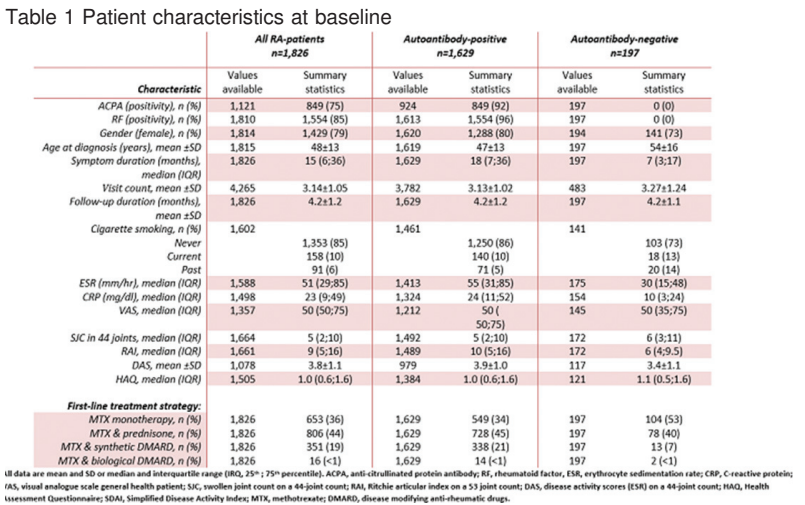

Conclusions: In conclusion, we found that autoantibody status was not associated with early remission in newly diagnosed RA-patients receiving methotrexate in real-world clinical practice. These results do not support the hypothesis that treatment should be tailored to "autoantibody status" when it comes to initiating methotrexate therapy as first-line anti-rheumatic treatment. Rather, our results indicate that that methotrexate is effective as primary anchor drug regardless of autoantibody status.

Disclosure of Interest: None declared

DOI: 10.1136/annrheumdis-2018-eular.4558

\section{FRI0644 THE USE OF MRI-DETECTED SYNOVITIS TO DETERMINE THE NUMBER OF INVOLVED JOINTS FOR THE 2010 ACR/EULAR CLASSIFICATION CRITERIA FOR RHEUMATOID ARTHRITIS - IS IT OF ADDITIONAL BENEFIT?}

A. C. Boer ${ }^{1,{ }^{*}}$, D. Boeters ${ }^{1}$, A. H. M. van der Helm - van Mil ${ }^{1,2} \cdot{ }^{1}$ Rheumatology, Leiden University Medical Center, Leiden, ${ }^{2}$ Rheumatology, Erasmus Medical Center, Rotterdam, Netherlands

Background: The 2010 ACR/EULAR classification criteria have been developed, as early classification of Rheumatoid Arthritis (RA) is important. The 2010criteria states that imaging can be used to determine the number of joints with synovitis. This seems reasonable as previous studies on Magnetic Resonance Imaging (MRI) in early arthritis patients have shown that synovitis is present in a substantial number of joints that were neither swollen nor tender at clinical examination. Although the development of the 2010-criteria was primarily data-driven, the suggestion to also use advanced imaging modalities to detect synovitis was based on expert opinion. Scientific data supporting the use of MRI is lacking.

Objectives: To assess the value of MRI-detected synovitis to determine the number of involved joints on the performance of the 2010-ACR/EULAR classification criteria for RA.

Methods: 277 consecutive patients with a clinical diagnosis of RA or undifferentiated arthritis (UA) were studied. They underwent contrast enhanced 1.5T MRI of MCP-, wrist- and MTP-joints at baseline. Two outcomes were studied after 1 year follow-up: disease modifying anti-rheumatic drugs (DMARD)-initiation and fulfilling the 1987-criteria. Test characteristics were calculated when the number of involved joints was determined with and without MRI-detected synovitis.

Results: At baseline, 143 of 277 patients did not fulfil the 2010-criteria when the number of involved joints was determined by clinical evaluation of swelling and tenderness. When MRI-detected synovitis was also considered 69 patients had increased joint counts. Of these, 36 patients received more points for the item 'number of involved joints' and 14 reached $\geq 6$ points and now fulfilled the 2010criteria for RA. Thus, $10 \%$ of patients that were formally classified as UA were additionally classified as having $R A$.

Without considering MRI-detected synovitis, the sensitivity of the 2010-criteria was $62 \%$ and the specificity $90 \%$, for DMARD initiation as outcome. With the addition of MRI-detected synovitis, the sensitivity increased to $67 \%$ and the specificity decreased to $84 \%$. The AUC changed from 0.76 to 0.75 . The net proportion of correctly reclassified patients was $-2.4 \%$. Of the additionally classified patients, $64 \%$ $(9 / 14)$ were started on DMARDs and were considered true positives, whereas $36 \%(5 / 14)$ were not treated with DMARDs and developed alternative clinical diagnoses during the first year.

Results for the outcome 1987-criteria fulfilment after 1-year were similar. The sensitivity changed from $79 \%$ to $81 \%$ and the specificity from $78 \%$ to $71 \%$ the proportion or correctly reclassified patents was $-5.1 \%$.
Conclusions: To our knowledge, this study is the first providing evidence on the value of MRI-detected synovitis in addition to tender and swollen joints for the classification of RA. We did not find an increased accuracy of the 2010 criteria when incorporating MRI-detected synovitis. Further research on this subject in other longitudinal cohorts is needed, but at present there is no scientific proof that MRI-detected synovitis is of additional benefit for classifying RA.

Disclosure of Interest: None declared DOI: 10.1136/annrheumdis-2018-eular.4501

\section{FRI0645 ANTI-DRUG ANTIBODIES TO CERTOLIZUMAB PEGOL ARE ASSOCIATED WITH LOW DRUG LEVELS AND REDUCED CLINICAL RESPONSE AT 3 MONTHS IN PATIENTS WITH INFLAMMATORY JOINT DISEASES. DATA FROM THE NOR-DMARD STUDY.}

J. E. Gehin ${ }^{1}$, G. L. Goll ${ }^{2}$, D. J. Warren ${ }^{1}$, S. W. Syversen ${ }^{2}$, J. Sexton ${ }^{2}$, E. K. Strand ${ }^{3}$, T. K. Kvien ${ }^{2}$, N. Bolstad ${ }^{1}$, E. Lie ${ }^{2}$. ${ }^{1}$ Department of Medical Biochemistry, Oslo University Hospital, ${ }^{2}$ Department of Rheumatology, Diakonhjemmet Hospital, Oslo, ${ }^{3}$ Lillehammer Hospital for Rheumatic Diseases, Lillehammer, Norway

Background: Anti-drug antibodies (ADAb) to biological drugs predispose patients to low drug levels and lack of treatment response. For certolizumab pego (CP) knowledge about the frequency and clinical relevance of ADAb is limited in patients with inflammatory joint diseases (IJD).

Objectives: To assess the frequency and clinical relevance of early ADAb development in patients with inflammatory joint diseases treated with CP.

Methods: Patients from the NOR-DMARD study $(n=310)$ with a clinical diagnosis of rheumatoid arthritis (RA, $n=91$ ), psoriatic arthritis (PsA, $n=61)$, axial spondyloarthritis (axSpA, $n=116)$ and other IJD (42) starting treatment with $\mathrm{CP}$, who had available biobank sample at 3 months follow-up, were included. Serum samples are non-trough samples collected at 3 months. Drug concentrations were analysed using an in-house immunofluorometric assay automated on the AutoDELFIA immunoassay platform. ADAb was detected by a principal assay measuring neutralising $A D A b$ and two confirmational tests (antigen-bridging test and a 3-step immunofluorometric assay). Patients with RA, PsA and axSpA were included in response analyses. Treatment response was defined by EULAR good/moderate response in RA, DAS28 improvement $>0.6$ in PsA, and ASDAS clinically important improvement (CII) in axSpA.

Results: After 3 months of treatment, 19 of $310(6.1 \%)$ patients were ADAb positive (5 RA, 4 PsA, 6 axSpA and 4 other IJD). ADAb positive patients had significantly lower CP levels than ADAb negative patients, median 1.0 (IQR $0.2-6.8$ ) vs 34.4 (IQR 21.2-44.7) mg/L $(P<0.001)$. Response data were available for 245 patients. Of these, only $1 / 11(9 \%)$ ADAb-positive patients was classified as a res ponder, while 10/11 (91\%) were non-responders. Among ADAb-negative patients with response data, 129/234 (55\%) were responders, while 105/234 (45\%) were non-responders.

Conclusions: ADAb against CP were detected in $6.1 \%$ of patients after 3 months of treatment and were associated with low drug levels and reduced treatment response. These results suggest that drug levels and ADAb may be important for monitoring efficacy of treatment with TNF inhibitors, but the clinical significance needs to be examined in randomised clinical strategy trials.

Disclosure of Interest: J. E. Gehin Consultant for: Roche, G. Goll Consultant for: Abbvie, Biogen, Boehringer Ingelheim, Orion Pharma, Eli Lilly, Novartis, Pfizer, MSD, Roche, UCB, D. Warren: None declared, S. Syversen Consultant for: Roche, J. Sexton: None declared, E. Strand Consultant for: Pfizer, T. Kvien Consultant for: AbbVie, Biogen, BMS, Boehringer Ingelheim, Celgene, Celltrion, Eli Lilly, Epirus, Hospira, Merck-Serono, MSD, Mundipharma, Novartis, Oktal, Orion Pharma, Hospira/Pfizer, Roche, Sandoz, UCB, N. Bolstad Consultant for: Pfizer, Orion Pharma, Napp pharmaceuticals, Takeda, Roche, E. Lie: None declared

DOI: 10.1136/annrheumdis-2018-eular.5257

\section{FRI0646 SYNOVIAL TISSUE HISTOPATHOLOGY FINDINGS IN EARLY RA. IS IT USEFULL? ANALYSIS OF THE BELGIAN CAP48 COHORT.}

C. Galant $^{1}$, L. Meric De Bellefon ${ }^{2}$, B. Lauwerys ${ }^{2}$, T. Sokolova $^{2}$, M. Stoenoiu $^{2}$, V. Badot ${ }^{3}$, A. Nzeusseu', F. Houssiau' ${ }^{2}$, P. Durez ${ }^{2}$. ${ }^{1}$ Pathology, ${ }^{2}$ Rheumatology, Cliniques universitaires Saint-Luc - Université Catholique de Louvain - Institut de Recherche Expérimentale et Clinique (IREC), ${ }^{3}$ Internal Medicine, Centre Hospitalier Universitaire Brugmann, Brussels, Belgium

Background: The development of ultrasound-guided synovial biopsy will enable synovial tissue collection from small joints and will facilitate histopathological studies, thus improving the understanding of early rheumatoid arthritis (ERA). The CAP48 cohort is an original multicentre prospective observational study of early 